\title{
Immuno-enhancement of Taishan Pinus massoniana pollen polysaccharides on recombinant Bordetella avium ompA expressed in Pichia pastoris
}

Liping Liu ${ }^{1}$, Cuilian $\mathrm{Yu}^{1}$, Chuanwen Wang, Mingxu Shao, Zhengui Yan, Xiaodong Jiang, Shanshan Chi, Zhen Wang, Kai Wei*, Ruiliang Zhu*

College of Animal Science and Technology, Shandong Agricultural University, Taian 271018, Shandong, China

*Corresponding author: Kai Wei, Ruiliang Zhu. College of Animal Science and Technology, Shandong Agricultural University, Taian 271018, Shandong, China.

Tel.: +86538 8242341; Fax: +86538 8242202.

E-mail address: weikaisdau@ 163.com (K. Wei); zhurl@ sdau.edu.cn (R. Zhu)

${ }^{1}$ These authors contributed equally to this work.

Running title: Novel Bordetella avium ompA subunit vaccine 
Abstract: Bordetellosis, caused by Bordetella avium (B. avium), continues to be an economic problem in the poultry industry of China. Vaccines with good protective ability are lacking. Thus, developing a novel vaccine against the $B$. avium infection is crucial. Here, we constructed a recombinant Pichia pastoris (P. pastoris) transformant capable of expressing the outer membrane protein A (ompA) of B. avium to prepare the recombinant ompA subunit vaccine and then evaluated its immune effects. To further investigate the immunomodulation effects of Taishan Pinus massoniana pollen polysaccharides (TPPPS) on this subunit vaccine, three concentrations $(20,40$, and 60 $\mathrm{mg} / \mathrm{mL}$ ) of TPPPS were used as the adjuvants of the ompA subunit vaccine respectively. The conventional Freund's incomplete adjuvant served as the control of TPPPS. Chickens in different groups were separately vaccinated with these vaccines thrice. During the monitoring period, serum antibody titers, concentrations of serum IL-4, percentages of CD4+ and CD8+ T-lymphocytes in the peripheral blood, lymphocyte transformation rate, and protection rate were detected. Results showed that the pure ompA vaccine induced the production of anti-ompA antibody, the secretion of IL-4, the increase of CD4+ T-lymphocytes counts and lymphocyte transformation rate in the peripheral blood. Moreover, the pure ompA vaccine provided a protection rate of $71.67 \%$ after the B. avium challenge. Notably, TPPPS adjuvant vaccines induced higher levels of immune responses than the pure ompA vaccine, and $60 \mathrm{mg} / \mathrm{mL}$ TPPPS adjuvant vaccine showed optimal immune effects and had a $91.67 \%$ protection rate. Our findings indicated that this recombinant B. avium 
ompA subunit vaccine combined with TPPPS had high immunostimulatory potential. Results provided a new perspective for $B$. avium subunit vaccine research.

Keywords: Bordetella avium, ompA, Pichia pastoris expression, subunit vaccine, TPPPS

\section{Introduction}

B. avium is the etiological agent of bordetellosis, which is clinically characterized by labored breathing, sneezing, and oculonasal discharges $[1,2]$. This pathogen has been widespread among avian species, such as Muscovy ducks, domesticated geese, partridges, turkeys, chickens, ostriches, cockatoos, conures, macaws, parrot finches, and cockatiels [3, 4]. In addition, it was also described in previous studies as an opportunistic human pathogen [5]. Because of its increasing secondary infection prevalence and slow growth rates, $B$. avium has brought significant economic losses in commercial flocks. Currently, the use of antibiotics in controlling diseases has not been recommended as they possess health hazards to consumers. Moreover, the live-attenuated vaccines and bacterin used to prevent bordetellosis cannot limit the infection and spread of $B$. avium, although they offer protection against severe diseases [6]. Therefore, developing a novel vaccine against $B$. avium infection is crucial.

OmpA, which is an important component of Gram-negative bacteria, has many active biological functions [7]. In our laboratory, the previous studies have shown that the ompA of B. avium expressed in Escherichia coli (E. coli) has certain immunogenicity [8]. Herein, to explore a novel subunit vaccine with better effects 
against B. avium, we intended to express the ompA using P. pastoris GS115 eukaryotic expression system. This system has been widely used in the expression of recombinant proteins because of its various advantages, such as easy cultivation, high yield, and capability to perform post-translational modifications [9]. Moreover, this expression system secretes few native proteins into the medium [10]. In the development of bacterial vaccines, the yeast-expressed antigen had been investigated in several animals and was proven to have good effects [11-13]. Therefore, the ompA expressed in $P$. pastoris GS115 may be a potential candidate in the development of a subunit vaccine against $B$. avium.

The application of adjuvant in vaccination is indispensable because adjuvant enhances the effects of vaccines. Adjuvant is believed to establish high and long-lasting immune responses by activating the innate immune system, thereby enhancing the adaptive immune response to an administered antigen $[14,15]$. Currently, plant-derived polysaccharides have attracted an increasing attention in the field of immune modulator [16]. As effective biological response modifiers, polysaccharides had been proven with excellent immuno-enhancement effects both in vivo and in vitro [17-19]. In our previous studies, we explored the polysaccharides from Aloe vera, Taishan Robinia pseudoacacia, and Firmiana simplex flower, and found that these polysaccharides possess immune enhancement function with different levels [20-22]. Notably, TPPPS, which we have been studying since 2003, has been proven as an effective adjuvant for the inactivated and subunit vaccines [23, 24]. However, little is known about the effects of TPPPS on the recombinant ompA 
protein based on eukaryotic expression system.

Therefore, to develop a novel $B$. avium vaccine, we constructed a recombinant $P$. pastoris transformant expressing B. avium ompA. Furthermore, to improve the effects of the prepared subunit vaccine, we used TPPPS as adjuvant. We evaluated the antibody production, cytokine secretion, $\mathrm{T}$ lymphocyte counts, lymphocyte transformation rate, and protection rate to assess the immune modulatory functions of TPPPS in chickens.

\section{Materials and methods}

\subsection{Ethics statement}

The animal procedures were approved by the Animal Care and Use Committee of Shandong Agricultural University (Permit number: 20010510), and performed in accordance with the "Guidelines for Experimental Animals" of the Ministry of Science and Technology (Beijing, China).

\subsection{Bacterial strains and plasmids}

B. avium strain LL was isolated from the sick chickens in 2008 (Shandong, China) and then preserved by our laboratory. The genetic homology of 16S rRNA between this B. avium strain LL and the reference strain S5 was 100\%. The B. avium strain was grown and maintained on lysogeny broth (LB) agar and broth at $37{ }^{\circ} \mathrm{C}$. P. pastoris GS115 and plasmid pPIC9 were purchased from Invitrogen (Carlsbad, CA, USA). The media were prepared according to the manuals of Pichia expression.

\subsection{Construction of recombinant transformant}

Based on the ompA gene sequence of B. avium (GeneBank accession number: 
M96550.1), a pair of primers (F: 5'-CCGGAATTCATGAACAAACCCTCCAAAAT3', the underlined bases encode an EcoR I restriction site, R: 5'-TTGCGGCCGCTTA ATGATGATGATGATGATGCTTGCGGCTACCGACGATT-3', the underlined bases encode a Not I restriction site) were designed to produce a $636 \mathrm{bp}$ fragment. The PCR product, which was confirmed by sequencing, was cloned into plasmid pPIC9, named pPIC9-ompA. This plasmid was transformed into a competent P. pastoris GS115 to obtain a transformant $P$. pastoris pPIC9-ompA in accordance with the manufacturer's instructions (Invitrogen). Blank plasmid pPIC9 was transformed into P. pastoris as a negative control.

\subsection{Expression, purification, and identification of B. avium ompA}

The methanol-induced expression of ompA in P. pastoris GS115 was performed following the manufacturer's instructions (Invitrogen). At 24, 48, 72, and $96 \mathrm{~h}$ post methanol induction, the culture supernatants of the $P$. pastoris transformed with the recombinant pPIC9-ompA plasmid and the P. pastoris transformed with blank pPIC9 plasmid were harvested through centrifugation, respectively. SDS-PAGE and Western blot analysis were carried out to identify the ompA as described in previous study [25] The mouse anti-omp polyclonal antibody used in western blot analysis was prepared by ourselves according to the previous method [26]. The recombinant protein ompA was purified using ProteinIso ${ }^{\mathrm{TM}}$ Ni-NTA Resin kit (TRANS, Beijing, China). Protein concentration was determined by Easy II Protein Quantitative Kit (BCA) (TRANS, Beijing, China).

\subsection{Preparation of vaccines}


TPPPS was provided by our laboratory and was prepared via hot water extraction and ethanol precipitation [24]. The purified recombinant ompA was mixed with TPPPS and Freund's incomplete adjuvant (Solarbio, Beijing) at a ratio of 1:1 respectively, reaching three final concentrations of 50,100 , and $150 \mu \mathrm{g} / \mathrm{mL}$. The concentration of TPPPS was set at three doses of 20,40 , and $60 \mathrm{mg} / \mathrm{mL}$ in three separate TPPPS adjuvant vaccines. The recombinant ompA mixed with TPPPS and Freund's incomplete adjuvant, were separately prepared to the corresponding adjuvant subunit vaccines.

\subsection{Animal experiment}

A total of 180 one-day-old SPF white leghorn chickens (male; Spirax Ferrer Poultry Co., Ltd, Jinan) were randomly divided into six sterilized isolators (groups I to VI), with 30 chickens in each. The ambient conditions were set at $20{ }^{\circ} \mathrm{C}$ to $25{ }^{\circ} \mathrm{C}$ and $30 \%$ to $40 \%$ relative humidity, and air entering the isolators was filtered. The chickens were allowed to acclimatize for three days before the start of the experiments.

Each chicken in groups I-VI was subcutaneously inoculated with $0.2 \mathrm{~mL}$ of the 20 , 40, and $60 \mathrm{mg} / \mathrm{mL}$ TPPPS adjuvant recombinant ompA vaccines, the Freund's incomplete adjuvant recombinant ompA vaccine, the pure recombinant ompA vaccine, and PBS respectively at 0,7 , and 14 days post the first vaccination (dpv). Groups I-VI were named OmpA-TPPPS (20), OmpA-TPPPS (40), OmpA-TPPPS (60), OmpA-Freund, OmpA, and PBS respectively. The final concentration of ompA in groups $\mathrm{I}-\mathrm{V}$ reached 50,100 , and $150 \mu \mathrm{g} / \mathrm{mL}$ at the first, second, and third vaccination 
respectively. At 3, 7, 14, 21, 28, 35, 42, and $49 \mathrm{dpv}$, three chickens from each group were selected randomly to determine the serum antibody titers, serum IL-4 concentrations, CD4+ and CD8+ T lymphocytes counts in the peripheral blood, and T-lymphocyte transformation rate. The chickens were not fed for $12 \mathrm{~h}$ before sampling.

One week after the third vaccination (at $21 \mathrm{dpv}$ ), 20 chickens from each group (except from the control group) were placed into a new isolator and challenged intranasally with $10 \mathrm{LD}_{50}$ B. avium LL strain. Clinical manifestation and survival status of the chickens were recorded for seven days after challenge. Clinical symptoms, including labored breathing, sneezing, and oculonasal discharges were monitored [1, 2]. The mortality, morbidity, and protection rate in each group were calculated according to the following formulas:

Mortality $(\%)=$ No. of dead chickens/Total No. $\times 100 \%$.

Morbidity $(\%)=$ No. of chickens with clinical symptoms/Total No. $\times 100 \%$.

Protective rate $(\%)=$ No. of chickens without clinical symptoms/Total No. $\times$ $100 \%$.

\subsection{Detection of serum antibody titers and IL-4 concentration}

At the sampling time, three blood samples $(1.0 \mathrm{~mL} /$ chicken $)$ from each group were collected randomly. The serum was separated through centrifugation and stored at $-20{ }^{\circ} \mathrm{C}$. The antibody titers were detected via indirect enzyme-linked immunosorbent assay (ELISA) as described previously [27]. The concentrations of serum IL-4 were detected using the chicken IL-4 ELISA kit (Langdun Bio-technology 
Co., Ltd., Shanghai) in accordance with the manufacturer's instructions. The absorbance was measured with a microplate reader at $450 \mathrm{~nm}$.

\subsection{Detection of CD4+ and CD8+ T lymphocyte counts in the peripheral blood}

Fresh anticoagulated peripheral bloods from three chickens $(1.0 \mathrm{~mL} / \mathrm{chicken})$ in each group were collected and mixed with $1.0 \mathrm{~mL}$ of PBS respectively. Each mixture was added to $2.0 \mathrm{~mL}$ of lymphocyte separation medium (Solarbio, China) to obtain lymphocytes after centrifugation as previously described [28]. Afterward, $10 \mu \mathrm{L}$ of CD4+ dye (Mouse Anti-chicken CD4-FITC, Cat: 8210-02, Lot: H4809-MM62, USA) and $10 \mu \mathrm{L}$ of CD8+ dye (Mouse Anti-chicken CD8-RPE, Cat: 8220-09, Lot: G149-VJ39K, USA) were added to $50 \mu \mathrm{L}$ of lymphocyte suspension. After incubation at $4{ }^{\circ} \mathrm{C}$ for $20 \mathrm{~min}$, the percentages of $\mathrm{CD} 4+$ and $\mathrm{CD} 8+\mathrm{T}$ lymphocytes were detected by flow cytometry (Guaga Easy Cyte Mini, USA).

\subsection{Peripheral blood lymphocyte proliferation}

The fresh anticoagulated peripheral bloods were separately collected from three chickens in each group, which were used to separate lymphocytes as previously described [28]. The lymphocytes were diluted by RPMI-1640 medium (Gibco, USA) containing $10 \% \mathrm{FBS}$, and the final density was adjusted to $1 \times 10^{6} / \mathrm{mL}$. These lymphocytes were then placed on 96-well culture plates at $100 \mu \mathrm{L}$ per well. Concanavalin A (ConA; Sigma, USA) was added to three wells of each row with the final concentration reaching $5 \mu \mathrm{g} / \mathrm{mL}$. Negative control (no ConA) wells were prepared. After incubation at $37 \quad{ }^{\circ} \mathrm{C}$ for $24 \quad \mathrm{~h}, \quad 10 \mu \mathrm{L}$ of 3-(4,5-dimethyl-2-thiazolyl)-2,5-diphenyl-2-H-tetrazolium bromide (MTT, $5 \mathrm{mg} / \mathrm{mL}$; 
Sigma, USA) was added to each well, and the solution was re-incubated for $4 \mathrm{~h}$. Then the supernatant was removed, and the absorbance at $490 \mathrm{~nm}$ was evaluated when the formazan crystals were dissolved for $5 \mathrm{~min}$ with $100 \mathrm{~mL}$ of DMSO in each well. The lymphocyte transformation rate (LTR) was calculated using the following formula: LTR $=($ ConA-stimulated mean - Non-stimulated mean $) /$ Control mean.

\subsection{Statistical analysis}

Data were expressed as mean $\pm \mathrm{SD}$, and Duncan's multiple-range test was performed to analyze the differences among groups using SPSS 17.0, where $p<0.05$ was considered statistically significant.

\section{Results}

\subsection{Expression of B. avium ompA in P. pastoris}

Firstly, the ompA gene of B. avium was amplified by PCR, and the PCR products were cloned in the expression vector pPIC9. After verification by gene sequencing (data not shown), the recombinant pPIC9-ompA plasmid was transformed into $P$. pastoris. Upon induction with methanol at different induction times, a novel protein band corresponding to $21 \mathrm{kDa}$ in the culture supernatant of the recombinant pPIC9-ompA transformant was observed by SDS-PAGE. The protein's molecular weight was identical to that of the foreign ompA, but this band did not appear in the culture supernatant of the control pPIC9 (blank plasmid) transformant (Fig. 1A). The protein was obviously detected in the supernatant at $48 \mathrm{~h}$ after cultivation, and the yield peaked at $72 \mathrm{~h}$ with a concentration of $16 \mathrm{mg} / \mathrm{L}$. After purification, only the target protein band with a molecular weight of $21 \mathrm{kDa}$ was observed in the result of 
SDS-PAGE (Fig. 1B). To determine the expression of the foreign protein, Western blot analysis was performed with the mouse anti-omp polyclonal antibody which was prepared by ourselves. After color development, only one band with a $21 \mathrm{kDa}$ molecular weight was observed (Fig. 1C), which corresponded to the band in SDS-PAGE, thereby indicating the existence of the recombinant ompA and its specific immune response characteristic.

\subsection{Changes of serum antibody titers}

The levels of antibody induced by vaccination are crucial in the examination of the effects of vaccines. The changes of serum antibody titers in each group are illustrated in Fig. 2. The antibody titers in all vaccine-inoculated groups were significantly higher than those in group PBS at 3-49 dpv $(p<0.05)$. At $28-49 \mathrm{dpv}$, groups OmpA-TPPPS (20), (40), (60), and OmpA-Freund had significantly higher antibody titers than that in group OmpA $(p<0.05)$. Notably, the antibody titers in groups inoculated with TPPPS adjuvant subunit vaccines were higher than that in group OmpA-Freund at 21, 35, and $42 \mathrm{dpv}(p<0.05)$. Moreover, compared with other groups, groups OmpA-TPPPS (40) and (60) had higher antibody titers at 21-42 dpv ( $p<0.05)$. A comparison between groups OmpA-TPPPS (40) and (60) revealed that the antibody titers in group OmpA-TPPPS (60) were higher than those in group OmpA-TPPPS (40), but the difference was not significant $(p>0.05)$. The results suggested that the recombinant protein ompA of B. avium had good immunogenicity, and the effects of TPPPS on promoting antibody titers were better than those of Freund's adjuvant. TPPPS at $60 \mathrm{mg} / \mathrm{mL}$ showed the strongest effect. 


\subsection{Changes of serum IL-4 concentration}

Cytokines involved in immune responses are crucial for fighting off infections [29]. The concentrations of serum IL-4 in each group are illustrated in Fig. 3. The IL-4 secretion in all vaccine-inoculated groups had an obvious increase relative to those of the PBS group, and the peak values were observed in groups OmpA-TPPPS (20), (40), (60), and OmpA-Freund at $28 \mathrm{dpv}$ and in group OmpA at $21 \mathrm{dpv}$. At 14-49 dpv, the concentrations of IL-4 in groups OmpA-TPPPS (20), (40), (60), and OmpA-Freund were significantly higher than those in group OmpA $(p<0.05)$. Notably, all groups inoculated with TPPPS adjuvant subunit vaccines had higher concentrations of IL-4 compared with group OmpA-Freund at 14, 21, 28, 35, and 49 dpv $(p<0.05)$. Furthermore, the concentrations of IL-4 in groups OmpA-TPPPS (40) and (60) were higher than those in group OmpA-TPPPS (20) at 21-49 dpv $(p<0.05)$, with group OmpA-TPPPS (60) having the highest concentrations of IL-4 ( $p>0.05)$. The results demonstrated that TPPPS significantly increased the secretion of serum IL-4 in chickens. TPPPS at $60 \mathrm{mg} / \mathrm{mL}$ showed the best performance.

\subsection{Changes of CD4+ and CD8+ T lymphocyte in the peripheral blood}

The percentages of $\mathrm{CD} 4+$ and $\mathrm{CD} 8+\mathrm{T}$ lymphocyte directly reflect the immune function of animals [30]. The changes of CD4+ and CD8+ T lymphocyte percentages in the peripheral blood of chickens are shown in Tables 1 and 2, respectively. The percentages of CD4+ in groups OmpA-TPPPS (20), (40), (60), and OmpA-Freund were significantly higher than those in group PBS at 3-49 dpv and in group OmpA at 14-49 dpv ( $p<0.05)$. Notably, at $14-42 \mathrm{dpv}$, the percentages of CD4+ in groups 
inoculated with TPPPS adjuvant subunit vaccines were significantly higher than those in group OmpA-Freund, while groups OmpA-TPPPS (40) and (60) had higher percentages of CD4+ than group OmpA-TPPPS $(20)(p<0.05)$. Furthermore, compared with group OmpA-TPPPS (40), group OmpA-TPPPS (60) had higher percentages of $\mathrm{CD} 4+$, but the difference was not significant $(p>0.05)$. However, the counts of CD8+ T lymphocytes did not present obvious difference compared with those of CD4+ T lymphocytes. These results suggested that TPPPS effectively improved the percentages of CD4+ instead of CD8+ T lymphocytes in the peripheral blood of chickens. The best effects were obtained with TPPPS at $60 \mathrm{mg} / \mathrm{mL}$.

\subsection{Changes of LTRs}

Phytoagglutinins, such as ConA, can non-specifically stimulate $\mathrm{T}$ mature lymphocytes in vitro, thereby leading to proliferation. The ratio of lymphocyte proliferation is a commonly used indicator to evaluate cellular immunity [31]. The changes in LTRs of chickens are shown in Table 3. At 14-49 dpv, the LTRs in groups OmpA-TPPPS (20), (40), (60), and OmpA-Freund were significantly higher than those in groups OmpA and PBS $(p<0.05)$. Notably, all groups inoculated with TPPPS adjuvant subunit vaccines had significantly higher LTRs than group OmpA-Freund at 7-49 dpv, and groups OmpA-TPPPS (40) and (60) had higher LTRs than group OmpA-TPPPS $(20)$ at $21-42 \mathrm{dpv}(p<0.05)$. Furthermore, the LTRs in group OmpA-TPPPS (60) were higher than those in group OmpA-TPPPS (40) but without significant difference. These results suggested that TPPPS effectively improved the LTRs in chickens, and the highest dose of TPPPS produced the optimal 
effects.

\subsection{Protective effects of ompA subunit vaccines}

To evaluate the protective effects of the ompA subunit vaccine against $B$. avium infection, 20 inoculated chickens from each group were challenged intranasally with $10 \mathrm{LD}_{50}$ B. avium LL strain at $21 \mathrm{dpv}$. Clinical symptoms and survival status of the chickens were recorded daily, and the results are illustrated in Fig. 4. After seven days observation, the morbidity and mortality of chickens vaccinated with the pure ompA vaccine were about $28 \%$ and $8 \%$ respectively. Although the two values in OmpA-Freund vaccine group were lower than those in pure ompA vaccine group, the differences were not statistically significant $(\mathrm{p}>0.05)$. However, the values in groups OmpA-TPPPS (40) and (60) were significantly lower than those in pure ompA vaccine group ( $\mathrm{p}<0.05$ ) (Fig. 4A and 4B). Additionally, the protection rates in groups OmpA-TPPPS (40) and (60) were also significantly higher than those in group OmpA ( $p<0.05)$, which the values of the former were both $91.67 \%$ and the latter was $71.67 \%$ (Fig. 4C). These data supported the immunogenicity of the yeast-expressed ompA to substantially protect chickens against $B$. avium infection, while the ompA subunit vaccine combined with 40 or $60 \mathrm{mg} / \mathrm{mL}$ of TPPPS could reach the optimal protection.

\section{Discussion}

Bordetellosis is a highly contagious upper respiratory disease that continues to bring negative effects on commercial flocks. Existing vaccines cannot prevent the infection and spread of $B$. avium. Here, we expressed a recombinant ompA of $B$. 
avium in P. pastoris and prepared a novel B. avium subunit vaccine. TPPPS was used as adjuvant to examine the immune enhancement effects of TPPPS on the subunit vaccine. We found that the pure ompA showed good immunogenicity, whereas TPPPS adjuvant enhanced the immune effects of subunit vaccine. The immune enhancing activity and safety of TPPPS have been demonstrated by our previous studies, and all of its enhancements on specific immune responses were based on the stimulation of a specific protein antigen $[23,24]$. Therefore, these enhanced immune effects were attributed to the specific stimulation of ompA antigen and the nonspecific promotion of the TPPPS adjuvant.

We used $P$. pastoris expression system to express the foreign ompA. This eukaryotic expression system has various advantages, such as easy cultivation, high yield, precise post-translational modifications, and little interference of native proteins $[9,10]$. In addition, this secretory expression system usually facilitates the subsequent purification process $[9,32]$, thereby avoiding damage to the content and activity of the recombinant ompA. Based on our results, the protein was detectable in the supernatant at $48 \mathrm{~h}$ post cultivation and reached the peak yield at $72 \mathrm{~h}$ post methanol induction with a concentration of $16 \mathrm{mg} / \mathrm{L}$. Thus, this foreign protein could be secreted in the medium with high quantity. Moreover, we observed that the amount of native proteins of $P$. pastoris secreted in the medium was very low, which greatly improved the purification efficiency of the recombinant ompA. These results indicated that the $P$. pastoris expression system was suitable for expressing the recombinant B. avium ompA. 
Humoral immunity response mediated by B lymphocytes is crucial in resisting infectious diseases. As an important indicator of the state of humoral immunity response, serum antibody titers of $B$. avium ompA were detected. Pure ompA subunit vaccine stimulated B lymphocytes into plasmocytes, thus secreted specific antibodies against B. avium, which proved that this $P$. pastoris-expressed protein had good immunogenicity. Moreover, the ompA subunit vaccine can protect chickens against $B$. avium infection with a protection rate of $71.67 \%$.

$\mathrm{T}$ lymphocyte has a key function in the immune response, and its proliferation is related to the status of cellular and humoral immunity in organisms [33]. The results showed that TPPPS significantly exhibited co-mitogenic activity on ConA-stimulated lymphocytes in vitro, which confirmed that TPPPS has an important function in improving $\mathrm{T}$ lymphocyte proliferation, thereby improving the levels of cellular and humoral immunity. In accordance with classical scheme of T-cell differentiation, mature CD4+ and CD8+ $\mathrm{T}$ lymphocytes in peripheral blood are regarded as two exclusive subsets. CD4+ T lymphocytes belong to $\mathrm{T}$ helper (Th) cells. The main function of these cells is to secrete cytokines and to induce and enhance immune response [34]. CD8+ T lymphocytes are cytotoxic $\mathrm{T}(\mathrm{Tc})$ cells and are responsible for the removal of target cells through direct killing effect [35]. In this study, we found that TPPPS increased the counts of CD4+ T lymphocytes, and the optimal effect was obtained at $60 \mathrm{mg} / \mathrm{mL}$ TPPPS. However, the counts of CD8+ T lymphocytes did not present any obvious difference relative to that of CD4+ T lymphocytes. These results suggested that the ompA subunit vaccine mainly induced Th immune response. 
For the secretion of cytokines, IL-4 is released by the activated T helper 2 (Th2) cells, which is crucial in promoting B lymphocytes proliferation. This Th2 cytokine also enhances the antigen presentation ability of B lymphocytes [36]. In the present study, OmpA-TPPPS subunit vaccine significantly increased the concentrations of serum IL-4. The increased production of IL-4 suggested the induction of the Th2 axis in response to TPPPS. These findings demonstrated that TPPPS can exercise their immune-regulatory functions via cytokine promotion.

Pinus massoniana pollen, treated as a traditional medicine for thousands of years in China, has revealed its excellent activitives in immune improvement. With the exploitation and development, Pinus massoniana pollen industry is forming a substantial scale. TPPPS, extracted from Taishan Pinus massoniana pollen, showed its great application prospects as a high-performance adjuvant. Recently, TPPPS has been demonstrated that it is composed of three kinds of polysaccharides (named TPPPS1-3), and each polysaccharide component was composed of different monosaccharides and showed different dominant activitives in anti-oxidant, anti-virus, and immunomodulation, indicating their synergistic effects on facilitating the immune function of organism [37]. Judging from this study, TPPPS used as adjuvant presented good effects on improving the immune response of the recombinant B. avium ompA subunit vaccine through improving serum antibody titers, concentrations of serum IL-4, percentages of CD4+ $\mathrm{T}$ lymphocytes in the peripheral blood, ratio of $\mathrm{T}$ lymphocyte proliferation and protection rate. Moreover, TPPPS had no toxic and 
side-effect on organisms and had no adverse effects on vaccines. TPPPS was a good choice as adjuvant for subunit vaccine.

In summary, the results demonstrated that the ompA subunit vaccine expressed in P. pastoris was capable of inducing humoral and cellular immune responses. Furthermore, adjuvant TPPPS presented good immune-enhancing effects on the subunit vaccine. Therefore, this study indicated the potential of the B. avium ompA subunit vaccine combined with TPPPS adjuvant in preventing $B$. avium infection. Our results provided a new perspective for development of the $B$. avium subunit vaccines.

\section{Acknowledgments}

This project was funded by the National Science Foundation of China (No. 31272595, 81541165), the Science and Technology Development Plan of Shandong Province (2012GNC11020), and the Chinese Medicine Antiviral Collaborative Innovation Center of Shandong Province Colleges (XTCX2014B0108).

\section{References}

[1] L.H. Arp, N.F. Cheville, Tracheal lesions in young turkeys infected with Bordetella avium, Am J Vet Res. 45 (1984) 2196-2200.

[2] Y.M. Saif, P.D. Moorhead, R.E. Whitmoyer, Scanning electron microscopy of tracheas from turkey poults infected with Alcaligenes faecalis, Avian Dis. 25 (1981) $730-735$.

[3] K.H. Hinz, G. Glunder, [Occurrence of Bordetella avium sp. nov. and Bordetella bronchiseptica in birds], Berl Munch Tierarztl Wochenschr. 98 (1985) 369-373. 
[4] T.R. Raffel, K.B. Register, S.A. Marks, L. Temple, Prevalence of Bordetella avium infection in selected wild and domesticated birds in the eastern USA, J Wildl Dis. 38 (2002) 40-46.

[5] A.T. Harrington, J.A. Castellanos, T.M. Ziedalski, J.E. Clarridge, 3rd, B.T. Cookson, Isolation of Bordetella avium and novel Bordetella strain from patients with respiratory disease, Emerg Infect Dis. 15 (2009) 72-74.

[6] R.B. Rimler, R.A. Kunkle, Bacterin-induced protection of turkeys against fowl cholera following infection with Bordetella avium, Avian Dis. 42 (1998) 752-756.

[7] S.G. Smith, V. Mahon, M.A. Lambert, R.P. Fagan, A molecular Swiss army knife: OmpA structure, function and expression, FEMS Microbiol Lett. 273 (2007) 1-11.

[8] X. Zhao, M. Liang, P. Yang, F. Guo, D. Pan, X. Huang, et al., Taishan Pinus massoniana pollen polysaccharides promote immune responses of recombinant Bordetella avium ompA in BALB/c mice, Int Immunopharmacol. 17 (2013) 793-798.

[9] J.L. Cereghino, J.M. Cregg, Heterologous protein expression in the methylotrophic yeast Pichia pastoris, FEMS Microbiol Rev. 24 (2000) 45-66.

[10] H. Aoki, M. Nazmul Ahsan, S. Watabe, Heterologous expression in Pichia pastoris and single-step purification of a cysteine proteinase from northern shrimp, Protein Expr Purif. 31 (2003) 213-221.

[11] E. Fingerut, B. Gutter, R. Meir, D. Eliahoo, J. Pitcovski, Vaccine and adjuvant activity of recombinant subunit B of E. coli enterotoxin produced in yeast, Vaccine. 23 (2005) 4685-4696. 
[12] R. Chen, Z. Zhou, Y. Cao, Y. Bai, B. Yao, High yield expression of an AHL-lactonase from Bacillus sp. B546 in Pichia pastoris and its application to reduce Aeromonas hydrophila mortality in aquaculture, Microb Cell Fact. 9 (2010) 39.

[13] D.D. Hartwig, T.L. Oliveira, F.K. Seixas, K.M. Forster, C. Rizzi, C.P. Hartleben, et al., High yield expression of leptospirosis vaccine candidates LigA and LipL32 in the methylotrophic yeast Pichia pastoris, Microb Cell Fact. 9 (2010) 98.

[14] D.T. O'Hagan, E. De Gregorio, The path to a successful vaccine adjuvant--'the long and winding road', Drug Discov Today. 14 (2009) 541-551.

[15] J.L. Virelizier, Adjuvants and formulations: How to make an immunogen from an antigen, Biologicals. 37 (2009) 139-140.

[16] P.V. Licciardi, J.R. Underwood, Plant-derived medicines: a novel class of immunological adjuvants, Int Immunopharmacol. 11 (2011) 390-398.

[17] Z.F. Zhang, G.Y. Lv, X. Jiang, J.H. Cheng, L.F. Fan, Extraction optimization and biological properties of a polysaccharide isolated from Gleoestereum incarnatum, Carbohydr Polym. 117 (2015) 185-191.

[18] Y. Fan, Y. Hu, D. Wang, Z. Guo, X. Zhao, L. Guo, et al., Epimedium polysaccharide and propolis flavone can synergistically stimulate lymphocyte proliferation in vitro and enhance the immune responses to ND vaccine in chickens, Int J Biol Macromol. 47 (2010) 87-92.

[19] J. Jiang, C. Wu, H. Gao, J. Song, H. Li, Effects of astragalus polysaccharides on immunologic function of erythrocyte in chickens infected with infectious bursa disease virus, Vaccine. 28 (2010) 5614-5616. 
[20] Z. Sun, K. Wei, Z. Yan, X. Zhu, X. Wang, H. Wang, et al., Effect of immunological enhancement of aloe polysaccharide on chickens immunized with Bordetella avium inactivated vaccine, Carbohyd Polym. 86 (2011) 684-690.

[21] M.F. Liang, G.H. Liu, Q.Y. Zhao, S.F. Yang, S.X. Zhong, G.L. Cui, et al., Effects of Taishan Robinia pseudoacacia Polysaccharides on immune function in chickens, Int Immunopharmacol. 15 (2013) 661-665.

[22] Z. Wang, X. Gao, Q. Zhao, C. Zhao, X. Zuo, R. Zhu, Antivirus and antibacterial effects of total flavones extracted from phoenix tree flower, Chinese Journal of Veterinary Science. 33 (2013) 272-276.

[23] G. Cui, S. Zhong, S. Yang, X. Zuo, M. Liang, J. Sun, et al., Effects of Taishan Pinus massoniana pollen polysaccharide on the subunit vaccine of Proteus mirabilis in birds, Int J Biol Macromol. 56 (2013) 94-98.

[24] K. Wei, Z. Sun, Z. Yan, Y. Tan, H. Wang, X. Zhu, et al., Effects of Taishan Pinus massoniana pollen polysaccharide on immune response of rabbit haemorrhagic disease tissue inactivated vaccine and on production performance of Rex rabbits, Vaccine. 29 (2011) 2530-2536.

[25] L.M. Temple, D.M. Miyamoto, M. Mehta, C.M. Capitini, S. Von Stetina, H.J. Barnes, et al., Identification and characterization of two Bordetella avium gene products required for hemagglutination, Infect Immun. 78 (2010) 2370-2376.

[26] X. Hu, R. Zhu, H. Liu, J. Bi, Study on extraction with supersonic and antigenicity of outer-membrane protein of Bordetella avium, Acta Microbiologica Sinica. 47 (2007) 714-717. 
[27] H. Denac, C. Moser, J.D. Tratschin, M.A. Hofmann, An indirect ELISA for the detection of antibodies against porcine reproductive and respiratory syndrome virus using recombinant nucleocapsid protein as antigen, J Virol Methods. 65 (1997) $169-181$.

[28] M. Mwanza, L. Kametler, A. Bonai, V. Rajli, M. Kovacs, M.F. Dutton, The cytotoxic effect of fumonisin B1 and ochratoxin A on human and pig lymphocytes using the Methyl Thiazol Tetrazolium (MTT) assay, Mycotoxin Res. 25 (2009) 233-238.

[29] S.F. Lowry, Cytokine mediators of immunity and inflammation, Arch Surg. 128 (1993) 1235-1241.

[30] C. Torti, M. Prosperi, D. Motta, S. Digiambenedetto, F. Maggiolo, G. Paraninfo, et al., Factors influencing the normalization of CD4+ T-cell count, percentage and CD4+/CD8+ T-cell ratio in HIV-infected patients on long-term suppressive antiretroviral therapy, Clin Microbiol Infect. 18 (2012) 449-458.

[31] G.L. Asherson, J. Ferluga, G. Janossy, Non-specific cytotoxicity by T cells activated with plant mitogens in vitro and the requirement for plant agents during the killing reaction, Clin Exp Immunol. 15 (1973) 573-589.

[32] Y. Luo, X. Kong, A. Xu, S. Jin, D. Wu, Expression, purification, and functional characterization of recombinant human interleukin-7, Protein expr purif. 63 (2009) $1-4$. 
[33] Y. Huang, C. Jiang, Y. Hu, X. Zhao, C. Shi, Y. Yu, et al., Immunoenhancement effect of rehmannia glutinosa polysaccharide on lymphocyte proliferation and dendritic cell, Carbohyd Polym. 96 (2013) 516-521.

[34] N. Olivares-Zavaleta, A. Carmody, R. Messer, W.M. Whitmire, H.D. Caldwell, Chlamydia pneumoniae inhibits activated human $\mathrm{T}$ lymphocyte proliferation by the induction of apoptotic and pyroptotic pathways, J Immunol. 186 (2011) 7120-7126.

[35] E.L. Pearce, M.C. Walsh, P.J. Cejas, G.M. Harms, H. Shen, L.S. Wang, et al., Enhancing CD8 T-cell memory by modulating fatty acid metabolism, Nature. 460 (2009) 103-107.

[36] T.R. Mosmann, R.L. Coffman, Heterogeneity of cytokine secretion patterns and functions of helper T cells, Adv immunol. 46 (1989) 111-147.

[37] S. Yang, K. Wei, F. Jia, X. Zhao, G. Cui, F. Guo, et al., Characterization and biological activity of Taishan Pinus massoniana pollen polysaccharide in vitro, PLOS ONE. 10 (2015) e0115638. 


\section{Figure legends}

Fig. 1. SDS-PAGE and Western blot identification of the expressed recombinant ompA. (A) SDS-PAGE identification of the recombinant ompA at different induction time. M, Pageruler pre-stained protein ladder; lane 1, the culture supernatant of $P$. pastoris transformed with blank pPIC9 vector at $96 \mathrm{~h}$ post methanol induction (negative control); lanes 2-5, the culture supernatant of $P$. pastoris transformed with the recombinant pPIC9-ompA plasmid at 24, 48, 72, and $96 \mathrm{~h}$ post methanol induction respectively. (B) Purification of the recombinant ompA. M, Pageruler pre-stained protein ladder; lane 1, the culture supernatant after column chromatography; lane 2, the purified recombinant ompA. (C) Western blot identification of the recombinant ompA with mouse anti-omp polyclonal antibody. M, Pageruler pre-stained protein ladder; lane 1, the culture supernatant of P. pastoris transformed with blank pPIC9 vector at $96 \mathrm{~h}$ post induction (negative control); lane 2, the culture supernatant of $P$. pastoris transformed with the recombinant pPIC9-ompA plasmid at $96 \mathrm{~h}$ post induction.

Fig. 2. Changes of serum antibody titers in experimental chickens. Chickens in six groups were vaccinated with $20 \mathrm{mg} / \mathrm{mL}$ (blue), $40 \mathrm{mg} / \mathrm{mL}$ (red), and $60 \mathrm{mg} / \mathrm{mL}$ (green) of TPPPS adjuvant ompA vaccines, Freund's adjuvant ompA vaccine (yellow), pure ompA vaccine (turquoise) and PBS (black) at 0,7 , and $14 \mathrm{dpv}$ respectively. Serum was collected at $3,7,14,21,28,35,42$, and $49 \mathrm{dpv}$. Then the antibody titers were determined by indirect ELISA. All values shown are means \pm SD from three independent experiments.

Fig. 3. Changes of serum IL-4 counts in experimental chickens. Chickens in six groups were vaccinated with $20 \mathrm{mg} / \mathrm{mL}$ (blue), $40 \mathrm{mg} / \mathrm{mL}$ (red), and $60 \mathrm{mg} / \mathrm{mL}$ 
(green) of TPPPS adjuvant ompA vaccines, Freund's adjuvant ompA vaccine (yellow), pure ompA vaccine (turquoise) and PBS (black) at 0,7 , and $14 \mathrm{dpv}$ respectively. Serum was collected at 3, 7, 14, 21, 28, 35, 42, and $49 \mathrm{dpv}$. Then serum IL-4 counts were detected by using the chicken IL-4 ELISA kits. All values shown are means \pm SD from three independent experiments.

Fig. 4. Morbility, mortadity and protection rate of chickens challenged with $B$. avium in different groups. Chickens in five groups were vaccinated with $20 \mathrm{mg} / \mathrm{mL}$ (blue), $40 \mathrm{mg} / \mathrm{mL}$ (red), and $60 \mathrm{mg} / \mathrm{mL}$ (green) of TPPPS adjuvant ompA vaccines, Freund's adjuvant ompA vaccine (yellow) and pure ompA vaccine (turquoise) at 0,7 , and $14 \mathrm{dpv}$ respectively. One week after the third vaccination, 20 chickens from each group were challenged intranasally with $10 \mathrm{LD}_{50}$ B. avium LL strain. Clinical symptoms and survival status were observed and recorded for seven successive days after challenge. 
A

B

C
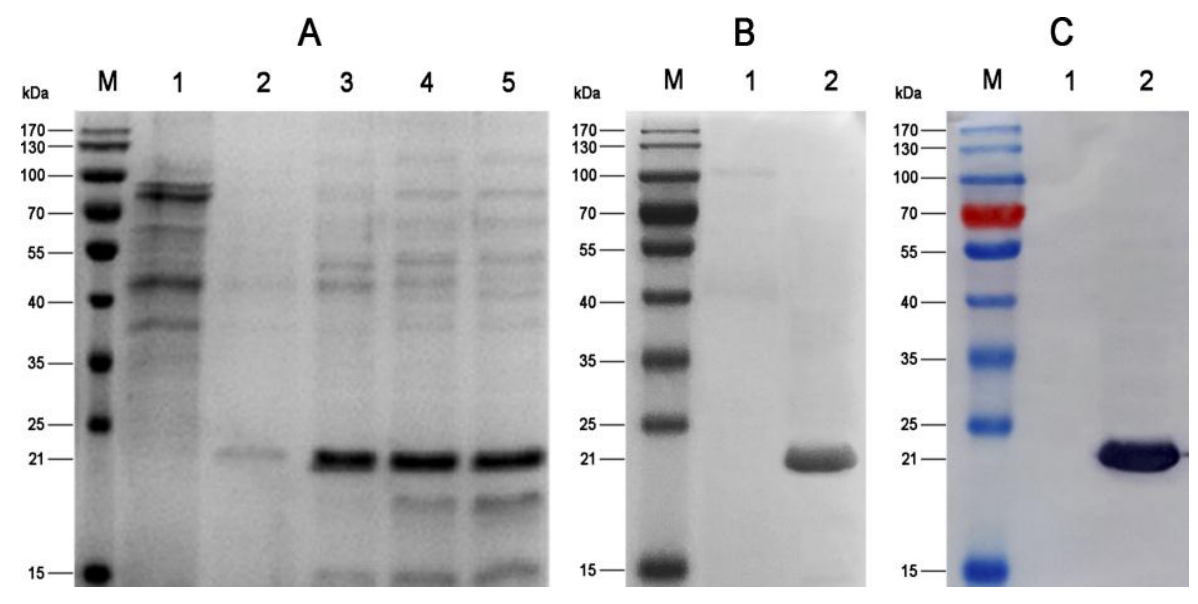


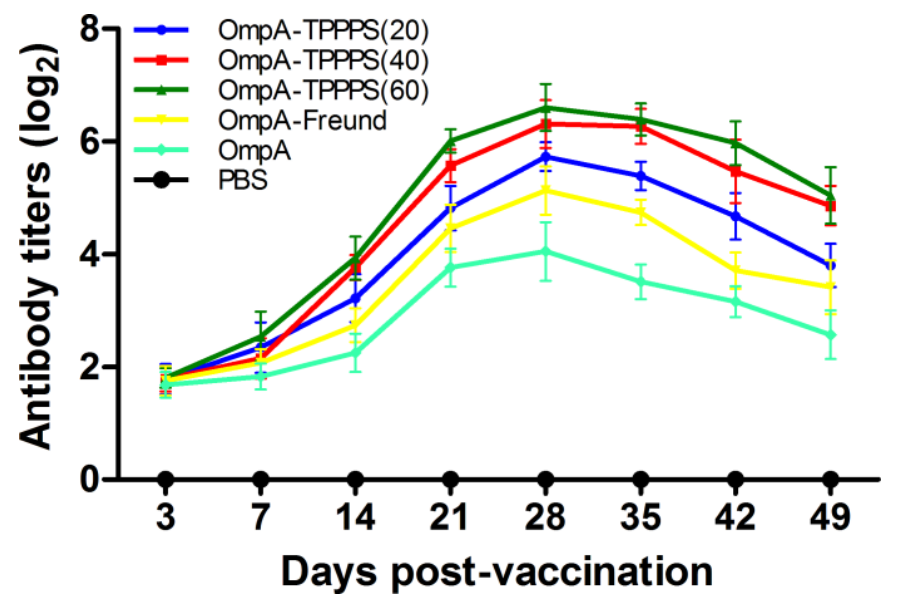




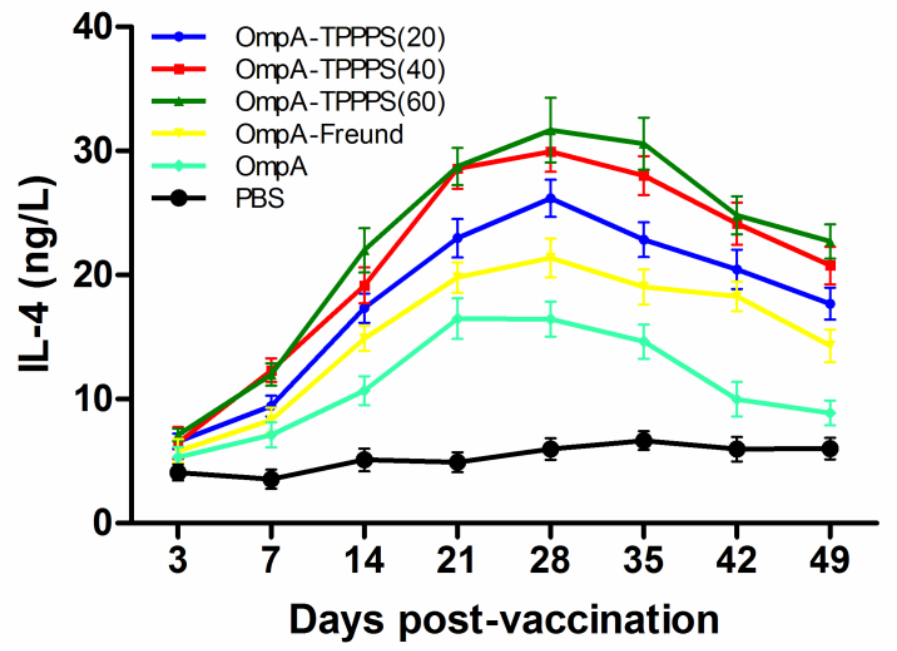


A

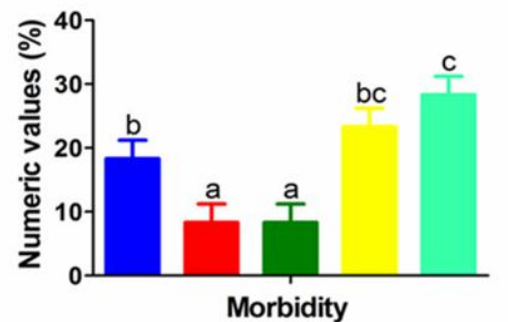

C

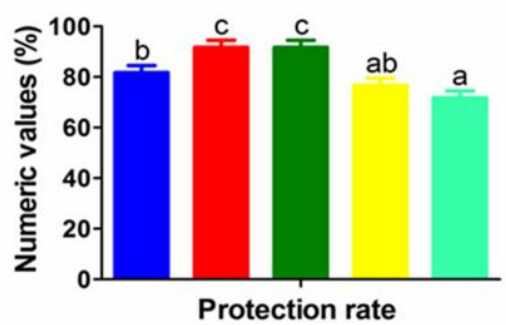

B

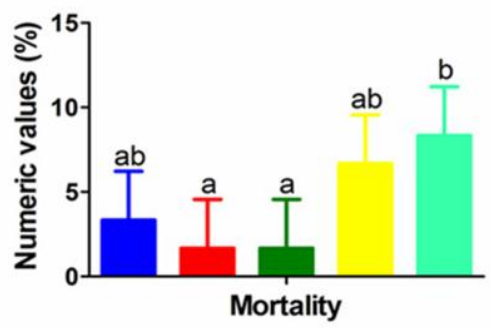

- OMPA-TPPPS(20)

OmpA-TPPPS(40)

$\square$ OmpA-TPPPS(60)

OmpA-Freund

OmpA 


\section{Table 1}

The changes of CD4+ T lymphocyte counts in the peripheral blood.

\begin{tabular}{|c|c|c|c|c|c|c|c|c|}
\hline \multirow{2}{*}{ Group $^{A}$} & \multicolumn{8}{|c|}{ Days post-vaccination ${ }^{\mathrm{B}}$} \\
\hline & 3 & 7 & 14 & 21 & 28 & 35 & 42 & 49 \\
\hline OmpA-TPPPS (20) & $33.54 \pm 0.41^{\mathrm{ab}}$ & $35.92 \pm 0.82^{\mathrm{b}}$ & $40.88 \pm 0.32^{\mathrm{c}}$ & $44.20 \pm 0.51^{\mathrm{b}}$ & $43.99 \pm 0.31^{\mathrm{b}}$ & $42.54 \pm 0.52^{\mathrm{c}}$ & $40.28 \pm 0.63^{\mathrm{b}}$ & $36.68 \pm 0.53^{\mathrm{bc}}$ \\
\hline OmpA-TPPPS (40) & $33.74 \pm 0.48^{\mathrm{ab}}$ & $38.02 \pm 0.62^{\mathrm{a}}$ & $42.24 \pm 0.56^{\mathrm{b}}$ & $46.56 \pm 0.34^{\mathrm{a}}$ & $47.93 \pm 0.61^{\mathrm{a}}$ & $44.32 \pm 0.55^{\mathrm{b}}$ & $42.07 \pm 0.68^{\mathrm{a}}$ & $37.96 \pm 0.87^{\mathrm{ab}}$ \\
\hline OmpA-TPPPS (60) & $34.11 \pm 0.48^{\mathrm{a}}$ & $38.03 \pm 0.98^{\mathrm{a}}$ & $43.82 \pm 1.03^{\mathrm{a}}$ & $46.53 \pm 0.54^{\mathrm{a}}$ & $48.09 \pm 0.47^{\mathrm{a}}$ & $46.31 \pm 0.81^{\mathrm{a}}$ & $42.46 \pm 0.71^{\mathrm{a}}$ & $38.89 \pm 0.69^{\mathrm{a}}$ \\
\hline OmpA-Freund & $32.99 \pm 0.57^{\mathrm{bc}}$ & $35.21 \pm 0.91^{\mathrm{bc}}$ & $38.70 \pm 0.64^{d}$ & $41.55 \pm 0.59^{\mathrm{c}}$ & $41.82 \pm 0.42^{\mathrm{c}}$ & $40.67 \pm 0.52^{\mathrm{d}}$ & $38.76 \pm 0.59^{c}$ & $36.28 \pm 0.75^{\mathrm{c}}$ \\
\hline OmpA & $32.54 \pm 0.47^{c}$ & $34.06 \pm 0.92^{\mathrm{c}}$ & $36.88 \pm 1.03^{\mathrm{e}}$ & $38.12 \pm 0.62^{\mathrm{d}}$ & $38.02 \pm 0.58^{d}$ & $37.07 \pm 0.62^{\mathrm{e}}$ & $35.85 \pm 0.61^{\mathrm{d}}$ & $34.22 \pm 0.60^{\mathrm{d}}$ \\
\hline PBS & $30.88 \pm 0.53^{\mathrm{d}}$ & $31.20 \pm 0.66^{\mathrm{d}}$ & $29.85 \pm 0.36^{\mathrm{f}}$ & $31.66 \pm 0.53^{\mathrm{e}}$ & $32.26 \pm 0.64^{\mathrm{e}}$ & $31.98 \pm 0.63^{f}$ & $32.64 \pm 0.65^{\mathrm{e}}$ & $32.29 \pm 0.83^{\mathrm{e}}$ \\
\hline
\end{tabular}

A Group names represent that chickens in these groups were vaccinated with $20 \mathrm{mg} / \mathrm{mL}, 40$ $\mathrm{mg} / \mathrm{mL}$, and $60 \mathrm{mg} / \mathrm{mL}$ of TPPPS adjuvant ompA vaccines, Freund's adjuvant ompA vaccine, pure ompA vaccine and PBS at 0,7 , and 14 dpv respectively.

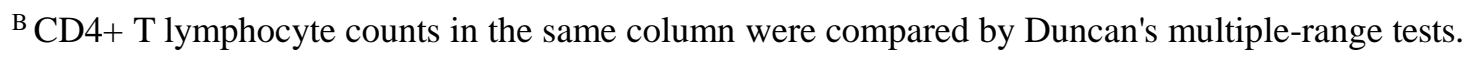
Different uppercase letter superscripts indicate significant differences $(p<0.05)$. Data are expressed as mean percentage $\pm \mathrm{SD}$. 
Table 2

The changes of $\mathrm{CD} 8+\mathrm{T}$ lymphocyte counts in the peripheral blood.

\begin{tabular}{|c|c|c|c|c|c|c|c|c|}
\hline \multirow{2}{*}{ Group $^{\mathrm{A}}$} & \multicolumn{8}{|c|}{ Days post-vaccination $^{B}$} \\
\hline & 3 & 7 & 14 & 21 & 28 & 35 & 42 & 49 \\
\hline OmpA-TPPPS (20) & $16.78 \pm 0.62^{\mathrm{a}}$ & $17.61 \pm 0.67^{\mathrm{a}}$ & $18.26 \pm 0.75^{\mathrm{a}}$ & $19.62 \pm 0.61^{\mathrm{ab}}$ & $19.66 \pm 0.72^{\mathrm{a}}$ & $18.93 \pm 0.55^{\mathrm{abc}}$ & $17.93 \pm 0.53^{\mathrm{abc}}$ & $17.75 \pm 0.47^{\mathrm{ab}}$ \\
\hline OmpA-TPPPS (40) & $16.84 \pm 0.52^{\mathrm{a}}$ & $17.80 \pm 0.57^{\mathrm{a}}$ & $18.33 \pm 0.55^{\mathrm{a}}$ & $19.71 \pm 0.68^{\mathrm{a}}$ & $20.04 \pm 0.60^{\mathrm{a}}$ & $19.40 \pm 0.53^{\mathrm{ab}}$ & $18.29 \pm 0.58^{\mathrm{ab}}$ & $18.05 \pm 0.89^{\mathrm{ab}}$ \\
\hline OmpA-TPPPS (60) & $16.87 \pm 0.40^{\mathrm{a}}$ & $17.72 \pm 0.59^{\mathrm{a}}$ & $18.62 \pm 0.52^{\mathrm{a}}$ & $20.02 \pm 0.53^{\mathrm{a}}$ & $20.56 \pm 0.53^{\mathrm{a}}$ & $19.76 \pm 0.52^{\mathrm{a}}$ & $18.80 \pm 0.38^{\mathrm{a}}$ & $18.74 \pm 0.64^{\mathrm{a}}$ \\
\hline OmpA-Freund & $16.81 \pm 0.47^{\mathrm{a}}$ & $17.40 \pm 0.57^{\mathrm{ab}}$ & $18.25 \pm 0.73^{\mathrm{a}}$ & $19.41 \pm 0.69^{\mathrm{ab}}$ & $19.65 \pm 0.70^{\mathrm{a}}$ & $18.67 \pm 0.45^{\mathrm{bc}}$ & $17.83 \pm 0.61^{\mathrm{abc}}$ & $17.67 \pm 0.69^{\mathrm{ab}}$ \\
\hline OmpA & $16.71 \pm 0.48^{\mathrm{a}}$ & $17.11 \pm 0.64^{\mathrm{ab}}$ & $17.91 \pm 0.61^{\mathrm{a}}$ & $18.51 \pm 0.62^{\mathrm{b}}$ & $18.01 \pm 0.74^{\mathrm{b}}$ & $17.95 \pm 0.58^{\mathrm{c}}$ & $17.47 \pm 0.52^{\mathrm{bc}}$ & $17.30 \pm 0.75^{\mathrm{b}}$ \\
\hline PBS & $16.20 \pm 0.59^{\mathrm{a}}$ & $16.35 \pm 0.73^{b}$ & $16.74 \pm 0.50^{\mathrm{b}}$ & $16.90 \pm 0.57^{\mathrm{c}}$ & $16.92 \pm 0.85^{\mathrm{b}}$ & $16.88 \pm 0.55^{\mathrm{d}}$ & $17.08 \pm 0.57^{\mathrm{c}}$ & $17.19 \pm 0.65^{\mathrm{b}}$ \\
\hline
\end{tabular}

${ }^{\mathrm{A}}$ Group names represent that chickens in these groups were were vaccinated with $20 \mathrm{mg} / \mathrm{mL}, 40$ $\mathrm{mg} / \mathrm{mL}$, and $60 \mathrm{mg} / \mathrm{mL}$ of TPPPS adjuvant ompA vaccines, Freund's adjuvant ompA vaccine, pure ompA vaccine and PBS at 0,7 , and $14 \mathrm{dpv}$ respectively.

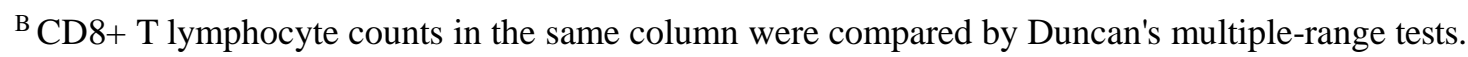
Different uppercase letter superscripts indicate significant differences $(p<0.05)$. Data are expressed as mean percentage $\pm \mathrm{SD}$. 
Table 3

The changes of $\mathrm{T}$ lymphocyte proliferation ratio in experimental chickens.

\begin{tabular}{|c|c|c|c|c|c|c|c|c|}
\hline \multirow{2}{*}{ Group $^{A}$} & \multicolumn{8}{|c|}{ Days post-vaccination $^{B}$} \\
\hline & 3 & 7 & 14 & 21 & 28 & 35 & 42 & 49 \\
\hline OmpA-TPPPS (20) & $22.14 \pm 0.63^{\mathrm{bc}}$ & $25.77 \pm 0.69^{b}$ & $29.60 \pm 0.65^{\mathrm{b}}$ & $34.24 \pm 0.96^{\mathrm{b}}$ & $34.09 \pm 1.12^{\mathrm{b}}$ & $32.58 \pm 0.74^{\mathrm{b}}$ & $31.46 \pm 0.67^{\mathrm{b}}$ & $27.11 \pm 0.95^{\mathrm{b}}$ \\
\hline OmpA-TPPPS (40) & $23.03 \pm 0.66^{\mathrm{ab}}$ & $25.46 \pm 0.87^{\mathrm{ab}}$ & $32.14 \pm 0.94^{\mathrm{b}}$ & $35.31 \pm 0.99^{a}$ & $36.26 \pm 1.03^{\mathrm{a}}$ & $35.83 \pm 0.93^{a}$ & $32.86 \pm 1.01^{\mathrm{a}}$ & $28.95 \pm 1.02^{\mathrm{ab}}$ \\
\hline OmpA-TPPPS (60) & $23.54 \pm 0.74^{\mathrm{a}}$ & $27.43 \pm 1.24^{\mathrm{a}}$ & $32.65 \pm 0.92^{\mathrm{a}}$ & $37.76 \pm 1.18^{a}$ & $36.94 \pm 1.18^{\mathrm{a}}$ & $36.87 \pm 0.79^{a}$ & $34.30 \pm 0.76^{\mathrm{a}}$ & $30.58 \pm 1.15^{\mathrm{a}}$ \\
\hline OmpA-Freund & $21.93 \pm 0.69^{\mathrm{bc}}$ & $24.24 \pm 0.74^{\mathrm{c}}$ & $27.65 \pm 0.62^{\mathrm{c}}$ & $31.42 \pm 1.24^{\mathrm{c}}$ & $31.38 \pm 1.02^{\mathrm{c}}$ & $30.79 \pm 0.72^{\mathrm{c}}$ & $28.98 \pm 0.78^{c}$ & $26.55 \pm 0.67^{c}$ \\
\hline OmpA & $21.17 \pm 0.73^{\mathrm{cd}}$ & $22.94 \pm 0.69^{c}$ & $25.18 \pm 0.51^{\mathrm{d}}$ & $28.29 \pm 0.71^{\mathrm{d}}$ & $26.35 \pm 0.84^{\mathrm{d}}$ & $26.24 \pm 0.97^{\mathrm{d}}$ & $25.45 \pm 0.79^{d}$ & $24.72 \pm 0.77^{\mathrm{d}}$ \\
\hline PBS & $20.55 \pm 0.60^{\mathrm{d}}$ & $21.09 \pm 0.65^{\mathrm{d}}$ & $22.07 \pm 1.09^{\mathrm{e}}$ & $23.09 \pm 0.84^{\mathrm{e}}$ & $23.24 \pm 0.88^{\mathrm{e}}$ & $23.82 \pm 0.83^{\mathrm{e}}$ & $23.64 \pm 0.73^{\mathrm{e}}$ & $24.69 \pm 0.62^{\mathrm{e}}$ \\
\hline
\end{tabular}

${ }^{\mathrm{A}}$ Group names represent that chickens in these groups were were vaccinated with $20 \mathrm{mg} / \mathrm{mL}, 40$ $\mathrm{mg} / \mathrm{mL}$, and $60 \mathrm{mg} / \mathrm{mL}$ of TPPPS adjuvant ompA vaccines, Freund' adjuvant ompA vaccine, pure ompA vaccine and PBS at 0,7 , and 14 dpv respectively.

B The ratio of $\mathrm{T}$ lymphocyte proliferation in the same column was compared by Duncan's multiple-range tests. Different uppercase letter superscripts indicate significant differences $(p<$ 0.05). Data are expressed as mean percentage $\pm \mathrm{SD}$. 